\title{
Ellipses Estimation from Their Digitization
}

\author{
Nataša Sladoje and Joviša Žunić \\ University of Novi Sad , Faculty of Engineering, \\ Trg D. Obradovica 6, 21000 Novi Sad, Yugoslavia \\ sladoje@uns.ns.ac.yu , ftn_zunic@uns.ns.ac.yu
}

\begin{abstract}
Ellipses in general position, and problems related to their reconstruction from digital data resulting from their digitization, are considered. If the ellipse

$$
E: \tilde{A}(x-p)^{2}+2 \tilde{B}(x-p)(y-q)+\tilde{C}(y-q)^{2} \leq 1, \tilde{A} \tilde{C}-\tilde{B}^{2}>0,
$$

is presented on digital picture of a given resolution, then the corresponding digital ellipse is:

$$
\begin{aligned}
D(E)= & \left\{(i, j) \mid A(i-a)^{2}+2 B(i-a)(j-b)+C(j-b)^{2} \leq r^{2},\right. \\
& i, j \text { are integers }\},
\end{aligned}
$$

where $r$ denotes the number of pixels per unit and $a=p r, b=q r$, $A=\tilde{A} r^{2}, B=\tilde{B} r^{2}, C=\tilde{C} r^{2}$.

Since the digitization of real shapes causes an inherent loss of information about the original objects, the precision of the original shape estimation from the corresponding digital data is limited, i.e. there is no possibility that the original ellipse can be recovered from the digital ellipse. What we present here is the estimation of parameters $A, B, C$ and center position $(a, b)$, of the ellipse digitized as above, with relative error bounded by $\mathcal{O}\left(\frac{1}{r^{13 / 11-\varepsilon}}\right)$ and absolute error bounded by $\mathcal{O}\left(\frac{1}{r^{4 / 11-z}}\right)$, (where $\varepsilon$ is an arbitrary positive number), that is, with the error tending to zero when the picture resolution increases. The obtained results imply that the half-axes of the original ellipse can be estimated with the same bounds of relative and absolute errors.
\end{abstract}

Index Terms - Pattern analysis, image processing, digital shapes, parameter estimation.

\section{Introduction}

Estimation of the relevant parameters of the original object, based on the data resulting from their digitization, is one of the the most important problems considered in computer vision and image processing ([2]). Digital shapes which appear the most often in practice are conic sections, including digital straight 
lines, (in the Euclidean plane) and so-called surfaces of the second order (in the Euclidean space). Some statistical parameter estimations of circular arcs can be founded in ([3]), bat the above mentioned problem, connected with the sets of digital points resulting from digitization of the ellipse in general position has not been solved by now. We find it might be interesting to give an efficient reconstruction of (general) ellipses by using digital data resulting from the digitization of the original object. Even though the problem of reconstructing corresponding digital ellipse still remains open, the method we describe provides obtaining the digital shape which can be considered as a good approximation of the coded one. For such purpose five properly chosen discrete moments are used.

The paper is organized as follows.

An asymptotical behaviour of discrete moments of digital ellipse, needed for the reconstruction and representation, is derived in the next section. An efficient estimation of the parameters of the original ellipse is given in Section 3. It is shown that the errors of such estimation tend to zero while $r$ tends to infinity, i.e. when the resolution of digital picture increases. The confirming experimental results are presented: even for $r=10$, relative deviation is shown to be less than $10 \%$ and for the absolute error we have 0.5 as the upper bound. For $r=100$, relative error is less than $0.6 \%$, and absolute error is bounded by 0.04 .

\section{Approximation of the Original Ellipse from its Digitization}

Consider an ellipse $E$ in the Euclidean plane, defined by the equation

$$
\tilde{A}(x-p)^{2}+2 \tilde{B}(x-p)(y-q)+\tilde{C}(y-q)^{2} \leq r^{2}, \tilde{A} \tilde{C}-\tilde{B}^{2}>0 .
$$

The ellipse $E$ is digitized by using digitizing method in which all the digital points (points with integer coordinates) in the ellipse are taken. The data,resulting from the digitization of a given ellipse $E$ obviously depend on the number of pixels per unit, i.e. on the picture resolution. Let $r$ denotes this number; then the set of digital points, defined by

$$
\begin{gathered}
D(E)=\left\{(i, j) \mid A(i-a)^{2}+2 B(i-a)(j-b)+C(j-b)^{2} \leq r^{2},\right. \\
i, j \text { are integers }\},
\end{gathered}
$$

is obtained as the digital picture of the ellipse $E\left(a=p r, b=q r, A=\tilde{A} r^{2}\right.$, $B=\tilde{B} r^{2}, C=\tilde{C} r^{2}$ ). This set will be considered as the digital ellipse.

As different ellipses may have identical digital images, there is some indispensable uncertainty in retrieving the original ellipse from its digital image. So, a natural and important question is how efficiently the original ellipse $E$ can be recovered from the data, resulting from its digitization $D(E)$ ? In this section it will be shown that certain information about digital ellipse $D(E)$ enables a reconstruction of $a, b, A, B$ and $C$, with error tending to zero while $r \rightarrow \infty$.

A $(k, l)$-moment, denoted by $m_{k, l}(S)$ for a shape $S$, in $2 D$ is defined by

$$
m_{k, l}(S)=\iint_{S} x^{k} y^{l} d x d y
$$


Considering that the parameters of the ellipse $E$ can easily be reconstructed if $m_{0,0}(E), m_{1,0}(E), m_{0,1}(E)$ and any two of $m_{2,0}(E), m_{1,1}(E)$ and $m_{0,2}(E)$ are known, and applying the analogous idea to the discrete shape, let us define the following integer parameters for a given ellipse $E$ :

- the number of points of $D(E)$, denoted by $R(E)$;

- the sum of $x$-coordinates of points of $D(E)$, denoted by $X(E)$;

- the sum of $y$-coordinates of points of $D(E)$, denoted by $Y(E)$;

- the sum of squares of $x$-coordinates of points of $D(E)$, denoted by $X X(E)$;

- the sum of squares of $y$-coordinates of points of $D(E)$, denoted by $Y Y(E)$.

Obviously, these parameters can easily be computed and are uniquely determined for any ellipse. They can be understood as its discrete moments. So, it is natural to introduce the following definition:

Definition 1 If a real ellipse $E$, given by $A(x-a)^{2}+2 B(x-a)(y-b)+C(y-b)^{2} \leq$ $r^{2}$, is digitized, and $R(E), X(E), Y(E), X X(E), Y Y(E)$ are calculated from $D(E)$, then the estimated values, $a_{e s t}, b_{e s t}, A_{e s t}, B_{e s t}$ and $C_{e s t}$, for the parameters of $E$ are

$$
\begin{aligned}
& \left(a_{e s t}, b_{e s t}\right)=\left(\frac{X(E)}{R(E)}, \frac{Y(E)}{R(E)}\right) \\
& A_{e s t}=\frac{4 \cdot r^{2} \cdot \pi^{2}}{(R(E))^{4}} \cdot\left(Y Y(E) \cdot R(E)-(Y(E))^{2}\right) ; \\
& B_{e s t}=\frac{4 \cdot r^{2} \cdot \pi}{(R(E))^{4}} . \\
& \quad \sqrt{16 \pi^{2}\left(X X(E) \cdot R(E)-(X(E))^{2}\right) \cdot\left(Y Y(E) \cdot R(E)-(Y(E))^{2}\right)-(R(E))^{6}} ; \\
& C_{e s t}=\frac{4 \cdot r^{2} \cdot \pi^{2}}{(R(E))^{4}} \cdot\left(X X(E) \cdot R(E)-(X(E))^{2}\right) .
\end{aligned}
$$

It can be expected that the ellipse defined by

$$
A_{e s t}\left(x-a_{e s t}\right)^{2}+2 B_{e s t}\left(x-a_{e s t}\right)\left(y-b_{e s t}\right)+C_{e s t}\left(y-b_{e s t}\right)^{2} \leq r^{2}
$$

is a good approximation of the original ellipse $E$. Its digitization, then, might be considered as a good approximation of $D(E)$.

In order to give the upper bound on the errors in estimating parameters of the original ellipse as defined above, we need asymptotical expressions for $R(E)$, $X(E), Y(E), X X(E)$ and $Y Y(E)$.

For convenience, in the rest of the paper it will be assumed that all the digital points have nonnegative coordinates. In other words, the origin is placed in the left-lower corner of the observed digital picture.

We cite the following result from the number theory ([1]).

Theorem 1 If $\mathcal{B}$ is a convex body in $R^{2}$, with $C^{3}$ boundary and positive curvature at every point of the boundary, then the number of lattice points belonging to $r \cdot \mathcal{B}$ is:

$$
R(r \cdot \mathcal{B})=r^{2} \cdot P(\mathcal{B})+\mathcal{O}\left(r^{\frac{7}{11}} \cdot(\log r)^{\frac{4 \pi}{22}}\right)
$$


where $P(\mathcal{B})$ denotes the area of $\mathcal{B}$, while $r \cdot \mathcal{B}$ is dilatation of $\mathcal{B}$ by factor $r$.

What we will use is weaker: $\quad R(r \cdot \mathcal{B})=r^{2} \cdot P(\mathcal{B})+\mathcal{O}\left(r^{\frac{7}{11}}+\varepsilon\right)$ for every $\varepsilon>0$. As a direct consequence is the asymptotical expression of $R(E)$ :

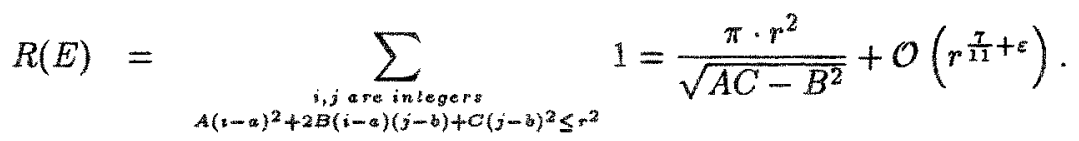

To give the asymptotical expressions for $X(E), Y(E), X X(E)$ and $Y Y(E)$, the following lemma will be needed.

Lemma 1 Let an ellipse $E$ be given by: $A(x-\alpha)^{2}+2 B(x-a)(y-b)+C(y-b)^{2} \leq$ $r^{2}$, and $E_{1}(i)$ and $E_{2}(i)$ be determined by

$E_{1}(i): \quad A(x-a)^{2}+2 B(x-a)(y-b)+C(y-b)^{2} \leq r^{2}$ and $x \leq i$, where $i$ is an integer and

$E_{2}(i): A(x-a)^{2}+2 B(x-a)(y-b)+C(y-b)^{2} \leq r^{2}$ and $x \geq i$, where $i$ is an integer.

Let $R(E), R\left(E_{1}(i)\right)$ and $R\left(E_{2}(i)\right)$ denote the numbers of digital points belonging to $E, E_{1}(i)$ and $E_{2}(i)$, respectively, while $P\left(E_{1}(i)\right)$ and $P\left(E_{2}(i)\right)$ denote the areas of $E_{1}$ and $E_{2}$, respectively. Then the following two (equivalent) relations

$$
\begin{aligned}
& R\left(E_{1}(i)\right)=P\left(E_{1}(i)\right)+\frac{1}{2} \cdot L\left(E_{1}(i)\right)+\mathcal{O}\left(r^{\frac{\tau}{11}+\varepsilon}\right) \text { and } \\
& R\left(E_{2}(i)\right)=P\left(E_{2}(i)\right)+\frac{1}{2} \cdot L\left(E_{2}(i)\right)+\mathcal{O}\left(r^{\frac{\tau}{11}+\varepsilon}\right)
\end{aligned}
$$

where

$$
L\left(E_{1}(i)\right)=L\left(E_{2}(i)\right)=\frac{\sqrt{B^{2}(i-a)^{2}-C\left(A(i-a)^{2}-r^{2}\right)}}{C}
$$

denotes the number of digital points of $D(E)$, lying on the line $x=i$, with the error bounded by 2, are satisfied.

Proof. The conditions of Theorem 1 can be relaxed to allow $\mathcal{B}$ having a finite number of corners, so (1) can be applied to the intersection of the interiors of two convex curves or, by subtraction, the region within one convex curve and outside another (for details, see [1]).

Let us consider the convex shape $\mathcal{L}(i)$, which is a subset of $E$, symmetrical with respect to the line $x=i$, and which contains all the digital points on the line $x=i$, within the ellipse $E$. Theorem 1 implies:

$$
\frac{1}{2} \cdot\left(R(\mathcal{L}(i))-L\left(E_{1}(i)\right)\right)=\frac{1}{2} \cdot\left(P(\mathcal{L}(i))-L\left(E_{1}(i)\right)\right)+\mathcal{O}\left(r^{7 / 11+\varepsilon}\right),
$$

where $R(\mathcal{L}(i))$ denotes the number of digital points belonging to $\mathcal{L}(i)$, while $P(\mathcal{L}(i))$ denotes the area of $\mathcal{L}(i)$.

If $E_{1}(i)$ denotes the (convex) shape $E_{1}(i) \cup \mathcal{L}(i)$, then, according to Theorem 1 , for the number of digital points belonging to $\tilde{E}_{1}(i)$, we have 
$R\left(E_{1}(i)\right)+\frac{1}{2} \cdot\left(R(\mathcal{L}(i))-L\left(E_{1}(i)\right)\right)=P\left(E_{1}(i)\right)+\frac{1}{2} \cdot P(\mathcal{L}(i))+\mathcal{O}\left(r^{7 / 11+\varepsilon}\right)$

Considering (3), the statement follows.

Now, the asymptotical expressions for $X(E)$ and $Y(E)$, can be given.

Theorem 2 Let the real numbers $a, b, A, B$ and $C$, satisfying $A C-B^{2}>0$, be given, and let all the values of $x$ and $y$, satisfying $A(x-a)^{2}+2 B(x-a)(y-b)+$ $C(y-b)^{2} \leq r^{2}$, be positive. Then the following asymptotical expressions hold:

$$
\begin{aligned}
& X(E)=\sum_{\substack{i, j a r e \text { integers } \\
A(i-a)^{2}+2 B(i-a)(j-b)+C(j-b)^{2} \leq r^{2}}} i=\frac{a \cdot \pi \cdot r^{2}}{\sqrt{A C-B^{2}}}+\mathcal{O}\left(a \cdot r^{\frac{\tau}{11}+\varepsilon}\right), \\
& Y(E)=\sum_{\substack{a, j a r e \text { integers } \\
A(i-a)^{2}+2 B(i-a)(j-b)+C(j-b)^{2} \leq r^{2}}} j=\frac{b \cdot \pi \cdot r^{2}}{\sqrt{A C-B^{2}}}+\mathcal{O}\left(b \cdot r^{\frac{7}{11}+\varepsilon}\right) .
\end{aligned}
$$

Proof. Let's notice that $X(E)$ is equal to the number of digital points belonging to $3 D$ body

$\mathcal{C}=\left\{(x, y, z) \mid A(x-a)^{2}+2 B(x-a)(y-b)+C(y-b)^{2} \leq r^{2}, \quad 0<z \leq x\right\}$.

If $a-\frac{r \sqrt{C}}{\sqrt{A C-B^{2}}}$ is denoted by $x_{\min }$, and $a+\frac{r \sqrt{C}}{\sqrt{A C-B^{2}}}$ is denoted by $x_{\max }$, then for the points $(x, y)$, satisfying $A(x-a)^{2}+2 B(x-a)(y-b)+C(y-b)^{2} \leq r^{2}$, we have $x \in\left[x_{\min }, x_{\max }\right]$.

Consider the number of digital points belonging to the body

$\mathcal{C}^{\prime}=\left\{(x, y, z) \mid A(x-a)^{2}+2 B(x-a)(y-b)+C(y-b)^{2} \leq r^{2}, x_{\min } \leq z \leq x\right\}$.

If $\mathcal{C}^{\prime}$ is intersected by planes $z=\left\lceil x_{\min }\right\rceil, z=\left\lceil x_{\min }\right\rceil+1, \ldots, z=\left\lfloor x_{\max }\right\rfloor$, than, obviously, each digital point from $\mathcal{C}^{\prime}$ belongs to one of those planes.

Expressing the volume of $\mathcal{C}^{\prime}$ as the sum of the volumes obtained by previous intersectings, we get

$$
\begin{aligned}
\operatorname{vol}\left(\mathcal{C}^{\prime}\right) & =\frac{a \cdot \pi \cdot r^{2}}{\sqrt{A C-B^{2}}}-x_{m i n} \cdot \frac{\pi \cdot r^{2}}{\sqrt{A C-B^{2}}} \\
& =\sum_{i=\left\lceil x_{\min }\right\rceil}^{\left\lfloor x_{\max }\right\rfloor-1} \operatorname{vol}\left(V_{i}\right)+\left(\left\lceil x_{m i n}\right\rceil-x_{m i n}\right) \cdot \frac{r^{2} \cdot \pi}{\sqrt{A C-B^{2}}}+\mathcal{O}(r),
\end{aligned}
$$

where

$$
\begin{aligned}
V_{i}= & \left\{(x, y, z) \mid A(x-a)^{2}+2 B(x-a)(y-b)+C(y-b)^{2} \leq r^{2},\right. \\
& x \geq i, \quad i \leq z<\min \{x, i+1\}\},
\end{aligned}
$$

for $i=\left\lceil x_{\min }\right\rceil,\left\lceil x_{\min }\right\rceil+1, \ldots,\left\lfloor x_{\max }\right\rfloor-1$.

Since $\operatorname{vol}\left(V_{i}\right)=P\left(E_{2}(i)\right)-\operatorname{vol}\left(V_{i}^{\prime}\right), \quad$ where

$$
\begin{aligned}
V_{i}^{\prime}= & \left\{(x, y, z) \mid A(x-a)^{2}+2 B(x-a)(y-b)+C(y-b)^{2} \leq r^{2},\right. \\
& x \geq i, \quad x \leq z<i+1\},
\end{aligned}
$$


and, obviously, $\quad \sum_{i=\left\lceil x_{\min }\right\rceil}^{\left\lfloor x_{\max }\right\rfloor-1} V_{i}^{\prime}=\frac{1}{2} \cdot \frac{\pi \cdot r^{2}}{\sqrt{A C-B^{2}}}+\mathcal{O}(r)$,

by applying Lemma 1, we obtain

$$
\begin{aligned}
\sum_{i=\left\lceil x_{m i n}\right\rceil}^{\left\lfloor x_{\max }\right\rfloor-1} \operatorname{vol}\left(V_{i}\right)= & \sum_{i=\left\lceil x_{\min }\right\rceil}^{\left\lfloor x_{\max }\right\rfloor-1}\left(R\left(E_{2}(i)\right)-\frac{1}{2} L\left(E_{2}(i)\right)+\mathcal{O}\left(r^{\frac{7}{1 I}+\varepsilon}\right)\right) \\
& -\frac{1}{2} \cdot \frac{\pi \cdot r^{2}}{\sqrt{A C-B^{2}}} .
\end{aligned}
$$

Further,

$$
\sum_{i=\left\lceil x_{\min }\right\rceil}^{\left\lfloor x_{\max }\right\rfloor-1} L\left(E_{2}(i)\right)=\frac{\pi \cdot r^{2}}{\sqrt{A C-B^{2}}}+\mathcal{O}(r)
$$

so (6) becomes

$$
\frac{a \cdot \pi \cdot r^{2}}{\sqrt{A C-B^{2}}}-x_{\min } \cdot \frac{\pi \cdot r^{2}}{\sqrt{A C-B^{2}}}=
$$

$=\sum_{i=\left\lceil x_{\min }\right\rceil}^{\left\lfloor x_{\max }\right\rfloor-1} R\left(E_{2}(i)\right)-\frac{\pi \cdot r^{2}}{\sqrt{A C-B^{2}}}+\left(\left\lceil x_{\min }\right\rceil-x_{\min }\right) \cdot \frac{r^{2} \cdot \pi}{\sqrt{A C-B^{2}}}+\mathcal{O}\left(r^{\frac{18}{11}+\varepsilon}\right)$

Finally, for the number of digital points in $C^{\prime}$, we have

$$
\sum_{i=\left\{x_{m i n}\right\rceil}^{\left\lfloor x_{m a x}\right\rfloor-1} R\left(E_{2}(i)\right)=\frac{a \cdot \pi \cdot r^{2}}{\sqrt{A C-B^{2}}}-\left(\left\lceil x_{m i n}\right\rceil-1\right) \frac{r^{2} \cdot \pi}{\sqrt{A C-B^{2}}}+\mathcal{O}\left(r^{\frac{18}{11}+\varepsilon}\right) .
$$

The number of digital points belonging to the body $C^{\prime \prime}$, determined by

$$
A(x-a)^{2}+2 B(x-a)(y-b)+C(y-b)^{2} \leq r^{2}, \quad 0<z<x_{\min },
$$

is equal to

$$
\left(\left\lceil x_{m i n}\right\rceil-1\right) \cdot \frac{\pi \cdot r^{2}}{\sqrt{A C-B^{2}}}+\mathcal{O}\left(a \cdot r^{\frac{7}{11}+\varepsilon}\right)
$$

(since $x_{\min }<a$ ), which, together with $(7)$, completes the proof. The proof of the expression (5) is analogous.

Theorem 3 Let the real numbers $a, b, A, B$ and $C$, satisfying $A C-B^{2}>0$, be given, and let all the values of $x$ and $y$, satisfying $A(x-a)^{2}+2 B(x-a)(y-b)+$ 
$C(y-b)^{2} \leq r^{2}$, be positive. Then the following asymptotical expressions hold:

$$
\begin{aligned}
X X(E) & =\sum_{\substack{i, j a r \in \text { integers } \\
A(\imath-a)^{2}+2 B(i-a)(j-b)+C(j-b)^{2} \leq r^{2}}} i^{2} \\
& =\frac{\pi \cdot r^{2}}{\sqrt{A C-B^{2}}}\left(a^{2}+\frac{C \cdot r^{2}}{4\left(A C-B^{2}\right)}\right)+\mathcal{O}\left(a^{2} r^{\frac{7}{12}+\varepsilon}\right), \\
Y Y(E) & =\sum_{\substack{i, j a r \in \text { integers } \\
A(n-a)^{2}+2 B(i-a)(j-b)+C(j-b)^{2} \leq r^{2}}} j^{2} \\
& =\frac{\pi \cdot r^{2}}{\sqrt{A C-B^{2}}}\left(b^{2}+\frac{A \cdot r^{2}}{4\left(A C-B^{2}\right)}\right)+\mathcal{O}\left(b^{2} r^{\frac{7}{11}+\varepsilon}\right) .
\end{aligned}
$$

Proof. Let's notice that $X X(E)$ is equal to the number of digital points belonging to $3 D$ body

$$
\mathcal{G}=\left\{(x, y, z) \mid A(x-a)^{2}+2 B(x-a)(y-b)+C(y-b)^{2} \leq r^{2}, \quad 0<z \leq x^{2}\right\} .
$$

As in the proof of Theorem 2, let $x \in\left[x_{\min }, x_{\max }\right]$ for the points $(x, y)$ satisfying $A(x-a)^{2}+2 B(x-a)(y-b)+C(y-b)^{2} \leq r^{2}$. Consider the number of digital points belonging to the body $\mathcal{G}^{\prime}$, given by

$$
\mathcal{G}^{\prime}: A(x-a)^{2}+2 B(x-a)(y-b)+C(y-b)^{2} \leq r^{2}, \quad\left(x_{\min }\right)^{2}<z \leq x^{2} .
$$

If $W_{i}$, for $i=\left\lceil x_{\min }\right\rceil,\left\lceil x_{\min }\right\rceil+1, \ldots,\left\lfloor x_{\max }\right\rfloor-1$, denotes the body

$$
\begin{gathered}
W_{i}=\left\{(x, y, z) \mid A(x-a)^{2}+2 B(x-a)(y-b)+C(y-b)^{2} \leq r^{2},\right. \\
\left.x \geq i, \quad i^{2}<z \leq \min \left\{x^{2},(i+1)^{2}\right\}\right\}
\end{gathered}
$$

then for the volume of $\mathcal{G}^{\prime}$ we have

$$
\begin{aligned}
\operatorname{vol}\left(\mathcal{G}^{\prime}\right) & =\frac{\pi \cdot r^{2}}{\sqrt{A C-B^{2}}} \cdot\left(a^{2}+\frac{C \cdot r^{2}}{4\left(A C-B^{2}\right)}\right)-\left(x_{m i n}\right)^{2} \cdot \frac{r^{2} \cdot \pi}{\sqrt{A C-B^{2}}} \\
& =\sum_{i=\left\{x_{m i n}\right\rceil}^{\left\lfloor x_{m a x}\right\rfloor-1} \operatorname{vol}\left(W_{i}\right)+\left(\left(\left\lceil x_{m i n}\right\rceil\right)^{2}-\left(x_{m i n}\right)^{2}\right) \cdot \frac{r^{2} \cdot \pi}{\sqrt{A C-B^{2}}}+\mathcal{O}(a) .
\end{aligned}
$$

Since

$$
\operatorname{vol}\left(W_{i}\right)=\left((i+1)^{2}-i^{2}\right) \cdot P\left(E_{2}(i)\right)-\operatorname{vol}\left(W_{i}^{\prime}\right)=(2 i+1) \cdot P\left(E_{2}(i)\right)-\operatorname{vol}\left(W_{i}^{\prime}\right)
$$

where

$$
\begin{aligned}
W_{i}^{\prime}= & \left\{(x, y, z) \mid A(x-a)^{2}+2 B(x-a)(y-b)+C(y-b)^{2} \leq r^{2},\right. \\
& \left.x \geq i, \quad x^{2}<z \leq(i+1)^{2}\right\}
\end{aligned}
$$


and

$$
\operatorname{vol}\left(W_{i}^{\prime}\right)=(2 i+1) \cdot \frac{1}{2} \cdot L\left(E_{2}(i)\right)+(2 i+1) \cdot \mathcal{O}\left(r^{\frac{1}{2}}\right)
$$

we have, according to Lemma 1, that

$$
\begin{aligned}
\sum_{i=\left\lceil x_{\min }\right\rceil}^{\left\lfloor x_{\max }\right\rfloor-1} \operatorname{vol}\left(W_{i}\right)= & \sum_{i=\left\lceil x_{m i n}\right\rceil}^{\left\lfloor x_{\max }\right\rfloor-1}(2 i+1) \cdot\left(R\left(E_{2}(i)\right)-L\left(E_{2}(i)\right)\right) \\
& +\sum_{i=\left\lceil x_{m i n}\right\rceil}^{\left\lfloor x_{\max }\right\rfloor-1}(2 i+1) \cdot \mathcal{O}\left(r^{\frac{7}{11}+\varepsilon}\right) .
\end{aligned}
$$

So, the volume of $\mathcal{G}^{\prime}$ can be expressed as

$$
\begin{aligned}
\operatorname{vol}\left(\mathcal{G}^{\prime}\right)= & \sum_{i=\left\lceil x_{m i n}\right\rceil}^{\left\lfloor x_{\max }\right\rfloor-1}(2 i+1) \cdot\left(R\left(E_{2}(i)\right)-L\left(E_{2}(i)\right)\right) \\
& \left.+\left(\left(\left\lceil x_{m i n}\right]\right)^{2}-\left(x_{m i n}\right)^{2}\right) \cdot \frac{\pi \cdot r^{2}}{\sqrt{A C-B^{2}}}+\mathcal{O}\left(a^{2} \cdot r^{\frac{7}{11}+\varepsilon}\right)\right) .
\end{aligned}
$$

Notice that

$$
\left.\sum_{i=\left\lceil x_{m i n}\right\rceil}^{\left\lfloor x_{m a x}\right\rfloor-1}(2 i+1) \cdot\left(R\left(E_{2}(i)\right)-L\left(E_{2}(i)\right)\right)+\mathcal{O}\left(a^{2} \cdot r^{\frac{7}{1 !}+\varepsilon}\right)\right)
$$

expresses the number of digital points in $\mathcal{G}^{\prime}$, excluding the points belonging to the plane $z=\left(\left\lceil x_{\min }\right\rceil\right)^{2}$.

Since the number of digital points belonging to

$$
\begin{aligned}
\mathcal{G}^{\prime \prime}= & \left\{(x, y, z) \mid A(x-a)^{2}+2 B(x-a)(y-b)+C(y-b)^{2} \leq r^{2},\right. \\
& \left.0<z \leq\left(\left\lceil x_{\min }\right\rceil\right)^{2}\right\}
\end{aligned}
$$

is equal to $\quad\left(\left\lceil x_{\min }\right\rceil\right)^{2} \cdot \frac{\pi \cdot r^{2}}{\sqrt{A C-B^{2}}}+\mathcal{O}\left(a^{2} \cdot r^{\frac{\pi}{11}+\varepsilon}\right) \quad$, the expression for the number of digital points in $\mathcal{G}=\mathcal{G}^{\prime} \cup \mathcal{G}^{\prime \prime}$ is

$$
\begin{aligned}
X X(E)= & \sum_{i=\left\lceil x_{\min }\right\rceil}^{\left\lfloor x_{\max }\right\rfloor-1}(2 i+1) \cdot\left(R\left(E_{2}(i)\right)-L\left(E_{2}(i)\right)\right)+\left(\left\lceil x_{\min }\right\rceil\right)^{2} \cdot \frac{\pi \cdot r^{2}}{\sqrt{A C-B^{2}}} \\
& +\mathcal{O}\left(a^{2} \cdot r^{\frac{7}{13}+\varepsilon}\right) .
\end{aligned}
$$

Then, by using expression for $\operatorname{vol}\left(\mathcal{G}^{\prime}\right)$, we have

$$
X X(E)=\frac{\pi \cdot r^{2}}{\sqrt{A C-B^{2}}} \cdot\left(a^{2}+\frac{C \cdot r^{2}}{4\left(A C-B^{2}\right)}\right)+\mathcal{O}\left(a^{2} \cdot r^{\frac{\gamma}{11}+\varepsilon}\right),
$$

which completes the proof. The proof of the expression (9) is analogous. 


\section{Errors in Estimating the Original Ellipse $E$ from its Discrete Moments $R(E), X(E), Y(E)$, $X X(E)$ and $Y Y(E)$}

Possible difference in the order of magnitude of $a, b$ and $r$, which is not excluded by the assumptions of Theorem 2, Theorem 3 and Theorem 4, affects the order of the error in estimating the parameters of the original ellipse as it is defined in Definition 1, if the asymptotical expressions for discrete moments $R(E), X(E)$, $Y(E), X X(E)$ and $Y Y(E)$ are directly substituted. However, as a consequence of the following statement, it can be assumed that $a, b$ and $r$ are of the same order.

Theorem 4 Let the ellipse $E=E(a, b, A, B, C): A(x-a)^{2}+2 B(x-a)(y-$ b) $+C(y-b)^{2} \leq r^{2}$ be given. Then the the values $X X(E) \cdot R(E)-(X(E))^{2}$, $Y Y(E) \cdot R(E)-(Y(E))^{2}, R(E), \frac{X(E)}{R(E)}-a$ and $\frac{Y(E)}{R(E)}-b$ are the constants with respect to a translation by a vector having integer components. In other words, the following equalities are satisfied:

a) $X X(E(a, b, A, B, C)) \cdot R(E(a, b, A, B, C))-(X(E(a, b, A, B, C)))^{2}=$ $=X X(E(a+k, b+l, A, B, C)) \cdot R(E(a+k, b+l, A, B, C))$

$-(X(E(a+k, b+l, A, B, C)))^{2}$,

b) $Y Y(E(a, b, A, B, C)) \cdot R(E(a, b, A, B, C))-(Y(E(a, b, A, B, C)))^{2}=$ $=Y Y(E(a+k, b+l, A, B, C)) \cdot R(E(a+k, b+l, A, B, C))$

$-(Y(E(a+k, b+l, A, B, C)))^{2}$,

c) $R(E(a, b, A, B, C))=R(E(a+k, b+l, A, B, C))$,

d) $\frac{X(E(a, b, A, B, C))}{R(E(a, b, A, B, C))}-a=\frac{X(E(a+k, b+l, A, B, C))}{R(E(a+k, b+l, A, B, C))}-(a+k)$,

e) $\frac{Y(E(a, b, A, B, C))}{R(E(a, b, A, B, C))}-b=\frac{Y(E(a+k, b+l, A, B, C))}{R(E(a+k, b+l, A, B, C))}-(b+l)$,

where $k$ and $l$ are integers.

Proof. a) For $k=1$ and $l=0$, the relation follows by using equalities

$$
\begin{gathered}
\sum_{(i, j) \in D(E(a+1, b, A, B, C))} 1=\sum_{(i, j) \in D(E(a, b, A, B, C))} 1 ; \\
\sum_{(i, j) \in D(E(a+1, b, A r, B r))} i=\sum_{(i, j) \in D(E(a, b, A, B, C))}(i+1),
\end{gathered}
$$

while other cases follow by induction or as a consequence of symmetry.

The proof of $b$ ) is analogous; the proofs of $c$ ), d) and e) are trivial.

Now, we give an upper bound on the precision of estimation the parameters of the ellipse from five discrete moments, $R(E), X(E), Y(E), X X(E)$ and $Y Y(E)$, corresponding to its digitization. 
Theorem 5 In estimating parameters of the ellipse $E$, given by $A(x-a)^{2}+$ $2 B(x-a)(y-b)+C(y-b)^{2} \leq r^{2}$ from its digitization $D(E)$, the relative errors

$$
\frac{a_{e s t}}{a}-1, \frac{b_{e s t}}{b}-1, \frac{A_{e s t}}{A}-1, \frac{B_{e s t}}{B}-1 \quad \text { and } \quad \frac{C_{e s t}}{C}-1
$$

are upper bounded by $\mathcal{O}\left(\frac{1}{r^{\frac{15}{11}}-\varepsilon}\right)$.

Proof. a) and b) follow directly from Definition 1 and (2)-(4). Since the same order of magnitude for $a, b$ and $r$ can be assumed, so $c$ ), d) and e) follow from Definition 1, (2)-(4), (7) and (8).

Numerical results are presented in Table 1.

It is not surprising that the relative errors tend to zero while $r$ tends to infinity, but convergence of the absolute errors to zero, with $r$ tending to infinity, might be unexpected. Anyway, the following results show that the absolute errors in estimating parameters of the digitized ellipse, just as the relative errors, tend to zero when the resolution of the digital picture is high.

Theorem 6 If the ellipse $E: A(x-a)^{2}+2 B(x-a)(y-b)+C(y-b)^{2} \leq r^{2}$ is digitized and its parameters are estimated from $D(E)$, then for the absolute errors we have

$$
a_{e s t}-a \text { and } b_{e s t}-b \text { are upper bounded by } \mathcal{O}\left(\frac{1}{\frac{1}{11}-\mathrm{c}}\right) \text {, while }
$$

$$
A_{e s t}-A, \quad B_{e s t}-B \text { and } C_{e s t}-C \text { are upper bounded by } \mathcal{O}\left(\frac{1}{r^{\frac{15}{11}}-*}\right) \text {. }
$$

Proof. By using Definition 1, the expressions for the appearing discrete moments and considering the assumption that $r, \alpha$ and $b$ have the same order of magnitude, the statement follows.

Table 2. contains the experimental results.

Since the slight change of the parameters $a, b, A, B$ and $C$ implies the slight change of the half-axes and the angle of rotation transforming given ellipse to the position where its axes are parallel to coordinate axes, which means that the slight change of $a, b, A, B$ and $C$ leads to the ellipse with slightly changed form and position, the digitization of the reconstructed ellipse can be considered as a good approximation of the coded one.

Moreover, we mention that the previous results imply that the half-axes of the original ellipse can be estimated with the same bound of the relative error as it is obtained for the center position, $(a, b)$, and parameters $A, B$ and $C$. Namely, if the half-axes of the original ellipse are denoted by $\tilde{A}$ and $\tilde{B}$, then the relations $\frac{1}{\tilde{A}^{2}}+\frac{1}{\bar{B}^{2}}=A+C$ and $\frac{1}{\tilde{A}^{2}} \cdot \frac{1}{\tilde{B}^{2}}=A C-B^{2}$ are satisfied, so $\frac{1}{\tilde{A}^{2}}$ and $\frac{1}{\hat{B}^{2}}$ can be obtained as the solution of the equation $x^{2}-(A+C) x+A C-B^{2}=0$. It is easy to conclude, then, that $\tilde{A}$ and $\tilde{B}$ can be recovered (by using $A_{\text {est }}, B_{\text {est }}$ 
and $\left.C_{e s t}\right)$ with the $\mathcal{O}\left(\frac{1}{r \frac{1}{15}-\varepsilon}\right)$ relative error. Similarly, it can be derived that the absolute errors of such estimation of the half-axes are bounded by $\mathcal{O}\left(\frac{1}{r \frac{t_{1}^{1}}{1 I}}\right)$.

Relative error in estimating the parameters $A, B, C$ and $(a, b)$ of the ellipse $E: A(x-a)^{2}+2 B(x-a)(y-b)+C(y-b)^{2} \leq r^{2}$, by $A_{e s t}, B_{e s t}, C_{e s t}$ and $\left(a_{e s t}, b_{e s t}\right)$, respectively; $M A X_{\text {rel_err }}=\max \left\{\left|\frac{A_{e s t}}{A}-1\right|,\left|\frac{B_{\text {est }}}{B}-1\right|,\left|\frac{C_{\text {est }}}{C}-1\right|,\left|\frac{a_{\text {est }}}{a}-1\right|,\left|\frac{b_{\text {est }}}{b}-1\right|\right\}$

\begin{tabular}{|c|c|c|c|c|c|c|}
\hline$A$ & $B$ & $C$ & $a$ & $\bar{b}$ & $r$ & $M A X_{\text {rel_err }}$ \\
\hline \multirow{3}{*}{7.5} & \multirow{3}{*}{5.2} & \multirow{3}{*}{11.7} & \multirow{3}{*}{57.7} & \multirow{3}{*}{76.6} & 10 & 0.08916 \\
\hline & & & & & 100 & 0.00297 \\
\hline & & & & & 1000 & 0.00032 \\
\hline \multirow{3}{*}{4.5} & \multirow{3}{*}{1.3} & \multirow{3}{*}{5.7} & \multirow{3}{*}{1615.9} & \multirow{3}{*}{849.6} & 10 & 0.05817 \\
\hline & & & & & 100 & 0.00519 \\
\hline & & & & & 1000 & 0.00011 \\
\hline \multirow{3}{*}{0.9} & \multirow{3}{*}{0.3} & \multirow{3}{*}{0.7} & \multirow{3}{*}{615.9} & \multirow{3}{*}{89.6} & 10 & 0.00602 \\
\hline & & & & & 100 & 0.00045 \\
\hline & & & & & 1000 & 0.00001 \\
\hline
\end{tabular}

Table 1.

Absolute error in estimating the parameters $A, B, C$ and $(a, b)$ of the ellipse $E: A(x-a)^{2}+2 B(x-a)(y-b)+C(y-b)^{2} \leq r^{2}$, by $A_{e s t}, B_{e s t}, C_{e s t}$ and $\left(a_{e s t}, b_{e s t}\right)$, respectively; $M A X_{a b s_{\_} e r r}=\max \left\{\left|A_{e s t}-A\right|,\left|B_{e s t}-B\right|,\left|C_{e s t}-C\right|,\left|a_{e s t}-a\right|,\left|b_{e s t}-b\right|\right\}$

\begin{tabular}{|c|c|c|c|c|c|c|}
\hline$A$ & $B$ & $\bar{C}$ & $a$ & $b$ & $r$ & $M A X_{a b s-e r r}$ \\
\hline \multirow{3}{*}{7.5} & \multirow{3}{*}{5.2} & \multirow{3}{*}{11.7} & \multirow{3}{*}{57.7} & \multirow{3}{*}{76.6} & 10 & 0.49874 \\
\hline & & & & & 100 & 0.02207 \\
\hline & & & & & 1000 & 0.01868 \\
\hline \multirow{3}{*}{4.5} & \multirow{3}{*}{1.3} & \multirow{3}{*}{5.7} & \multirow{3}{*}{1615.9} & \multirow{3}{*}{849.6} & 10 & 0.21307 \\
\hline & & & & & 100 & 0.03291 \\
\hline & & & & & 1000 & 0.01272 \\
\hline \multirow{3}{*}{0.9} & \multirow{3}{*}{0.3} & \multirow{3}{*}{0.7} & \multirow{3}{*}{615.9} & \multirow{3}{*}{89.6} & 10 & 0.03488 \\
\hline & & & & & 100 & 0.02942 \\
\hline & & & & & 1000 & 0.00311 \\
\hline
\end{tabular}

Table 2. 


\section{Comments and Conclusion}

Since the ellipses appear very often in practice of computer vision and image processing, the solution of the problem of their efficient reconstruction from the corresponding digital picture might be useful. Mentioning that the digitization of real shapes causes an inherent loss of information about the original objects, which implies that the precision of the estimation of the original shape from the corresponding digital data is limited, in previous sections we presented a constant time reconstruction of the parameters in the equation of the original ellipse with $\mathcal{O}\left(\frac{1}{r \frac{13}{11}-c}\right)$-relative error and $\mathcal{O}\left(\frac{1}{r \frac{3}{11}-\varepsilon}\right)$-absolute error, where $\varepsilon$ is an arbitrary positive number, and $r$ is the number of pixels per unit, which means that the errors of such reconstruction tend to zero when the the picture resolution is high. Consequently, the half-axes of the original ellipse can be reconstructed from the estimated values of the parameters $A, B$ and $C$, with the errors (both, relative and absolute) of the same order as the parameters, themselves. As a result, the coded digital ellipse can be approximated by the digitization of the reconstructed ellipse. Five discrete moments, corresponding to the digitization of the original ellipse, are needed for that purpose.

\section{References}

[1] M. N. Huxley, "Exponential Sums and Lattice Points", Proc. London Math. Soc. (3), 60, 471-502, 1990.

[2] J. Koplowitz and A. Bruckstein "Design of Perimeter Estimators for Digitized Planar Shapes", IEEE Trans. Pattern Analysis and Machine Intelligence, vol. 11, pp. 611-622, 1989.

[3] M. Worring and A.W.M. Smeulders, "Digitized circular arcs: characterization and parameter estimation", IEEE Trans. PAMI, vol. 17, pp. 587-598, 1995 . 